\title{
Echocardiographic evidence of left ventricular untwisting-filling interplay
}

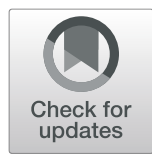

\author{
Amir Hodzic ${ }^{1,2,3^{*}}$ D, Damien Garcia ${ }^{4}$, Eric Saloux ${ }^{2}$, Paula A. B. Ribeiro ${ }^{3}$, Amélie Ethier ${ }^{3}$, James D. Thomas ${ }^{5}$, \\ Paul Milliez ${ }^{2}$, Hervé Normand ${ }^{1}$ and Francois Tournoux ${ }^{3}$
}

\begin{abstract}
Background: Left ventricular untwisting generates an early diastolic intraventricular pressure gradient (DIVPG) than can be quantified by echocardiography. We sought to confirm the quantitative relationship between peak untwisting rate and peak DIVPG in a large adult population.

Methods: From our echocardiographic database, we retrieved all the echocardiograms with a normal left ventricular ejection fraction, for whom color Doppler M-Mode interrogation of mitral inflow was available, and left ventricular untwisting rate was measurable using speckle tracking. Standard indices of left ventricular early diastolic function were assessed by Doppler (peaks $E, e^{\prime}$ and Vp) and speckle tracking (peak strain rate Esr). Load dependency of DIVPG and untwisting rate was evaluated using a passive leg raising maneuver.

Results: We included 154 subjects, aged between 18 to 77 years old, 63\% were male. Test-retest reliability for color Doppler-derived DIVPG measurements was good, the intraclass correlation coefficients were 0.97 [0.91-0.99] and 0.97 [0.67-0.99] for intra- and inter-observer reproducibility, respectively. Peak DIVPG was positively correlated with peak untwisting rate $(r=0.73, P<0.001)$. On multivariate analysis, peak DIVPG was the only diastolic parameter that was independently associated with untwisting rate. Age and gender were the clinical predictive factors for peak untwisting rate, whereas only age was independently associated with peak DIVPG. Untwisting rate and DIVPG were both load-dependent, without affecting their relationship.
\end{abstract}

Conclusions: Color Doppler-derived peak DIVPG was quantitatively and independently associated with peak untwisting rate. It thus provides a reliable flow-based index of early left ventricular diastolic function.

Keywords: Diastolic function, Untwisting rate, Color Doppler M-mode, Intraventricular pressure gradient

\section{Introduction}

Left ventricular (LV) untwisting is a key component of diastolic function [1]. This untwisting motion of the LV on its longitudinal axis occurs with elastic recoil of the base and apex from their previous systolic positions. The untwisting generates a diastolic intraventricular pressure gradient (DIVPG), contributing to the suction of blood from the left atrium into the LV at low atrial pressure [2]. Over the last decade, the assessment of LV untwisting motion and DIVPGs has been of increasing interest in cardiac imaging since they were independently

\footnotetext{
* Correspondence: hodzic-a@chu-caen.fr

'Department of Clinical Physiology, INSERM COMETE, Normandie Univ, UNICAEN, CHU de Caen Normandie, 14000 Caen, France

2Department of Cardiology, Normandie Univ, UNICAEN, CHU de Caen

Normandie, 14000 Caen, France

Full list of author information is available at the end of the article
}

associated with LV relaxation and restoring forces [3-5]. Tissue Doppler and later speckle tracking were used to measure LV untwisting. Although it was initially considered promising when its performance and accuracy were compared to those obtained with reference methods like cardiac magnetic resonance imaging and sonomicrometry $[6,7]$, its use is not widespread in clinical practice because of technical challenges and poor reproducibility $[8,9]$. Color Doppler M-mode echocardiography is a validated technique for non-invasive assessment of DIVPGs $[10,11]$. This approach estimating the instantaneous local pressure gradient from the measure of the spatiotemporal distribution of blood velocities along the LV long axis could provide an overview of the LV diastolic function. Prior studies in large animals and small human cohorts have suggested a close physiological relationship

(c) The Author(s). 2020 Open Access This article is distributed under the terms of the Creative Commons Attribution 4.0 International License (http://creativecommons.org/licenses/by/4.0/), which permits unrestricted use, distribution, and 
between those two parameters of early diastole [12, 13]. However, Doppler-derived DIVPG has not been widely applied in the LV diastolic functional assessment. We were able to develop fully automated software to measure non-invasively DIVPGs. The objective of this study was to investigate in a large population of subjects without heart disease the quantitative relationship between peak DIVPG assessed by color Doppler M-mode and peak LV untwisting rate estimated by speckle tracking.

\section{Methods}

The echocardiographic laboratory of the CHUM (Centre Hospitalier de l'Université de Montréal) has a research database in which healthy volunteers and patients have consented to participate, authorizing access to their clinical information. We retrospectively retrieved from this database all echocardiograms performed over one year and meeting the following criteria: 1) an LV ejection fraction $(\mathrm{LVEF}) \geq 50 \%, 2)$ an available color M-Mode tracing in apical four-chamber view as usually acquired for the assessment of the propagation flow velocity, and 3) the appropriate short-axis views to measure the untwisting rate using speckle tracking. Studies with the following criteria were excluded: known history of cardiac disease, regional wall motion abnormality, moderate or severe valvular heart disease, atrial fibrillation, or paced rhythm. Cardiovascular risk factors, physical activity behavior, and medical history were abstracted from either the questionnaire linked to our research database or from their medical records. The transthoracic echocardiograms were performed using one of our commercially available ultrasound machines: Vivid E9, Vivid 7 or Vivid q (GE Medical System, Milwaukee, WI, USA), equipped with a 2.5 $\mathrm{MHz}$ transducer. Acquisitions were performed in left lateral decubitus during an apnoea on three consecutive cardiac cycles with a stable ECG signal. All measurements were archived offline on a remote workstation (EchoPAC version 112, GE, Horten, Norway).

A passive leg raising maneuver had been performed by lifting the subject's legs to a $45^{\circ}$ angle to assess the load dependency of DIVPG and untwisting rate measurements, evaluated during the maneuver after heart rate stabilization. Lifting the legs passively from the horizontal position induces a gravitational transfer of blood from the lower limbs toward the intrathoracic compartment resulting in increased cardiac preload.

\section{Routine echocardiographic analysis}

LV function was evaluated according to the current guidelines of the American Society of Echocardiography $[9,14]$. LVEF was calculated using the Biplane Modified Simpson's method. LV dimensions and mass were measured from a parasternal long-axis view and normalized to body surface area. Left atrial (LA) volume was measured by the Biplane method of disks during endsystole and normalized to body surface area. LV diastolic function was analyzed by measuring peak early $(E)$ and late (A) transmitral Doppler velocities, peak mitral annulus early velocity (e') by tissue Doppler imaging (average of septal and lateral peaks), and color M-mode Doppler flow propagation velocity $(\mathrm{Vp})$ with the slope of the Nyquist velocity isoline after adjusting the color Doppler baseline. The E/A, E/e' and E/Vp ratios were calculated. Doppler parameters were obtained as the average value of three consecutive cardiac cycles.

\section{Speckle tracking imaging and left ventricular untwisting analysis}

LV untwisting rate was measured by two-dimensional speckle tracking on the parasternal short-axis views at the basal (mitral valve leaflets visible) and the apical (circular LV cavity, with no papillary muscle visible) levels, as previously described [7]. To ensure acceptable image quality, the frame rate was between 50 to $80 \mathrm{~Hz}$. LV rotation was measured by displaying the rotation angle against time during the cardiac cycle in the apical and basal short-axis views. The averaged LV rotation and rotational velocity were obtained from six segments of each short-axis view, on three consecutive cardiac cycles. Peak untwisting rate (in $\mathrm{deg} / \mathrm{s}$ ) was defined from the rotation velocity waveform as the first negative peak occurring during isovolumetric relaxation (Fig. 1). Speckle tracking was also used to measure the systolic peak of LV global longitudinal strain (GLS), and the peak of global early diastolic strain rate (Esr) obtained from each of the three apical views.

\section{DIVPG assessment by color Doppler M-mode}

The non-invasive estimation of instantaneous DIVPGs by color Doppler M-Mode has been validated in animal and human studies $[10,11]$. The investigators simplified the intracardiac pressure-velocity relationship by assuming that gravitational potential energy and energy losses due to viscous dissipation are both negligible. If the M-mode scanline approximates a flow streamline, it turns out that the Euler momentum equation yields the spatiotemporal pressure-velocity relationship along the M-line:

$$
\frac{\partial P}{\partial s}=-\rho\left[\frac{\partial v}{\partial t}+v \frac{\partial v}{\partial s}\right]
$$

where $\mathrm{P}(\mathrm{s}, \mathrm{t})$ and $\mathrm{v}(\mathrm{s}, \mathrm{t})$ stands for pressure and Doppler velocity, respectively. The coordinate $s$ represents the affine (spatial) coordinate along the streamline, and $t$ is time. Density $\rho$ is constant since blood is incompressible in the investigated conditions. This simplified equation shows that the pressure gradient $(\partial \mathrm{P} / \partial \mathrm{s})$ is the result of 

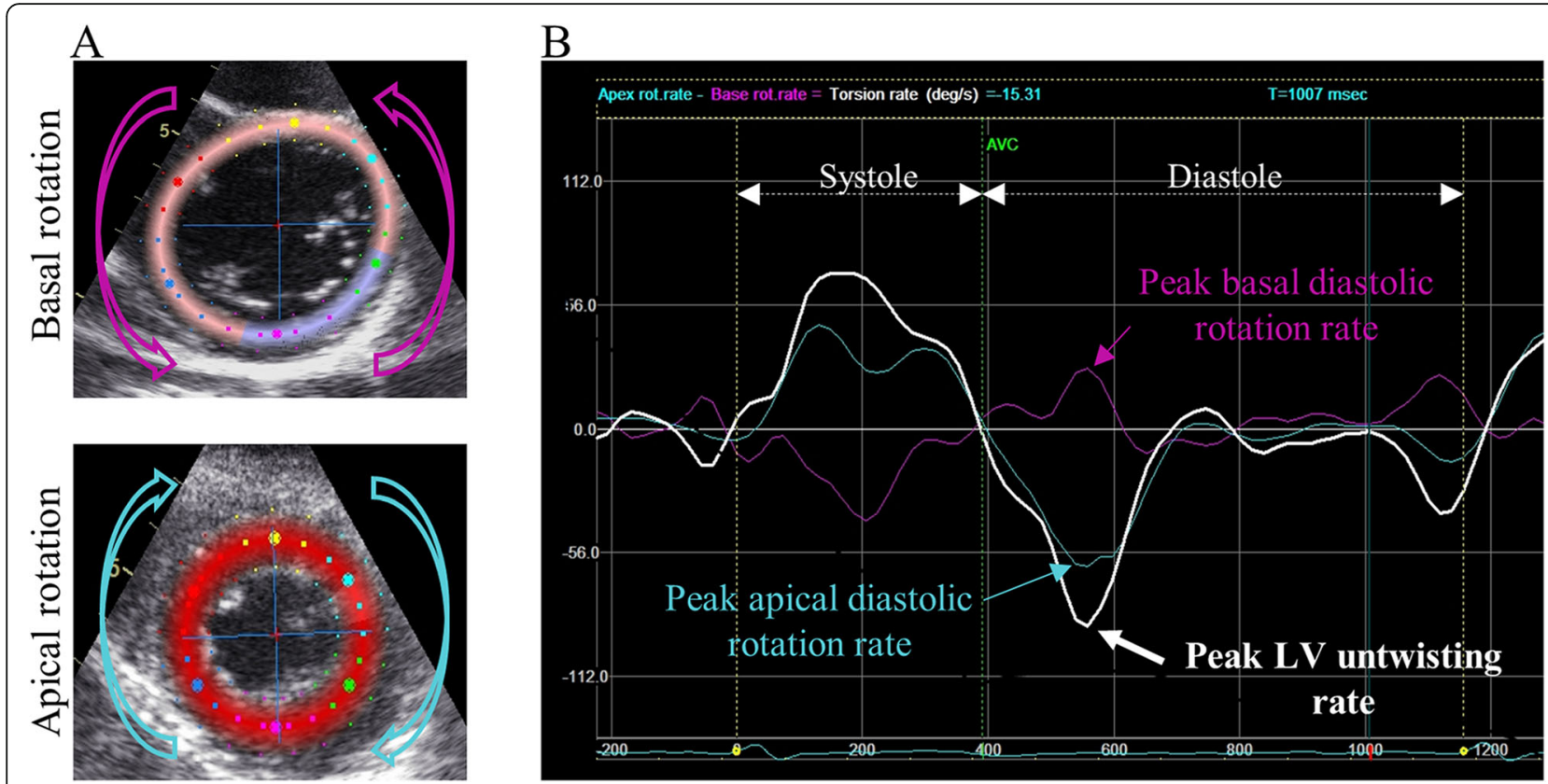

Fig. 1 Measurement of left ventricular untwisting rate using speckle trackinga: Speckle tracking of left ventricular rotation in the short-axis view, at the basal and the apical levels (the arrows indicate the untwisting direction). $\mathbf{b}$ : Rotation rate curves from speckle tracking analysis.

inertial $(\partial \mathrm{v} / \partial \mathrm{t})$ and convective $(\partial \mathrm{v} / \partial \mathrm{s})$ components [15]. Integration of this equation along the M-line, from the LV base to apex, yields an estimation of the DIVPG expressed by the unsteady Bernoulli equation:

$$
P_{\text {base }}-P_{\text {apex }}=\frac{1}{2} \rho\left(v_{\text {apex }}^{2}-v_{\text {base }}^{2}\right)+\rho \int_{\text {base }}^{a p e x} \frac{\partial v}{\partial t} d s
$$

Of note, this equation was successfully used and validated in aortic stenosis to estimate the instantaneous transvalvular pressure difference [16]. Based on the unsteady Bernoulli equation, all the color Doppler M-Mode tracings acquired along the base-to-apex axis using a standard apical four-chamber view could be used for our study. Three consecutive cardiac cycles were collected. The raw DICOM color Doppler MMode images were transferred to hierarchical h5 data files using EchoPAC. The images were then processed off-line using our homemade Matlab program to estimate the instantaneous pressure difference between the LV base and apex. The basal and apical levels were located manually by the operator. The numerical algorithm was then fully automatic and included unsupervised dealiasing and smoothing [17, 18] to ensure high inter-operator reproducibility. The peak pressure differences were determined during early filling for three successive heart cycles and averaged. The flowchart in Fig. 2 illustrates the sequence of pre- and post-processing used to assess peak DIVPG.

\section{Statistical analysis}

Statistical analysis was performed with MedCalc Statistical Software (version 13.2.0, MedCalc Software, Belgium). The results were expressed as mean \pm standard deviation if normally distributed, if not, as median \pm interquartile range. Linear regression analyses were used to study the relationships between echocardiographic and clinical data. A multivariate linear regression analysis was used to determine the potential independent predictors of peak untwisting rate and peak DIVPG. The multivariate model was developed with stepwise inclusion and exclusion at a significant level of 0.1. The measurements during load changes were analyzed using a paired sample t-test. Test-retest reproducibility of DIVPG acquisitions was determined on two independent color Doppler M-mode measures made by the same operator for intra-observer variability, and by two independent operators on the same subject for interobserver variability. We used the intraclass correlation coefficient with a 95\% confidence interval and the absolute difference between repeated measurements expressed in percent of their mean. The level of significance was defined as $P<0.05$.

\section{Results}

Population characteristics

Among 223 subjects meeting the inclusion and exclusion criteria, DIVPG and LV untwisting rate analyses were both feasible in 154/223 (69\%) subjects included in the study. Exclusions were mainly due to 


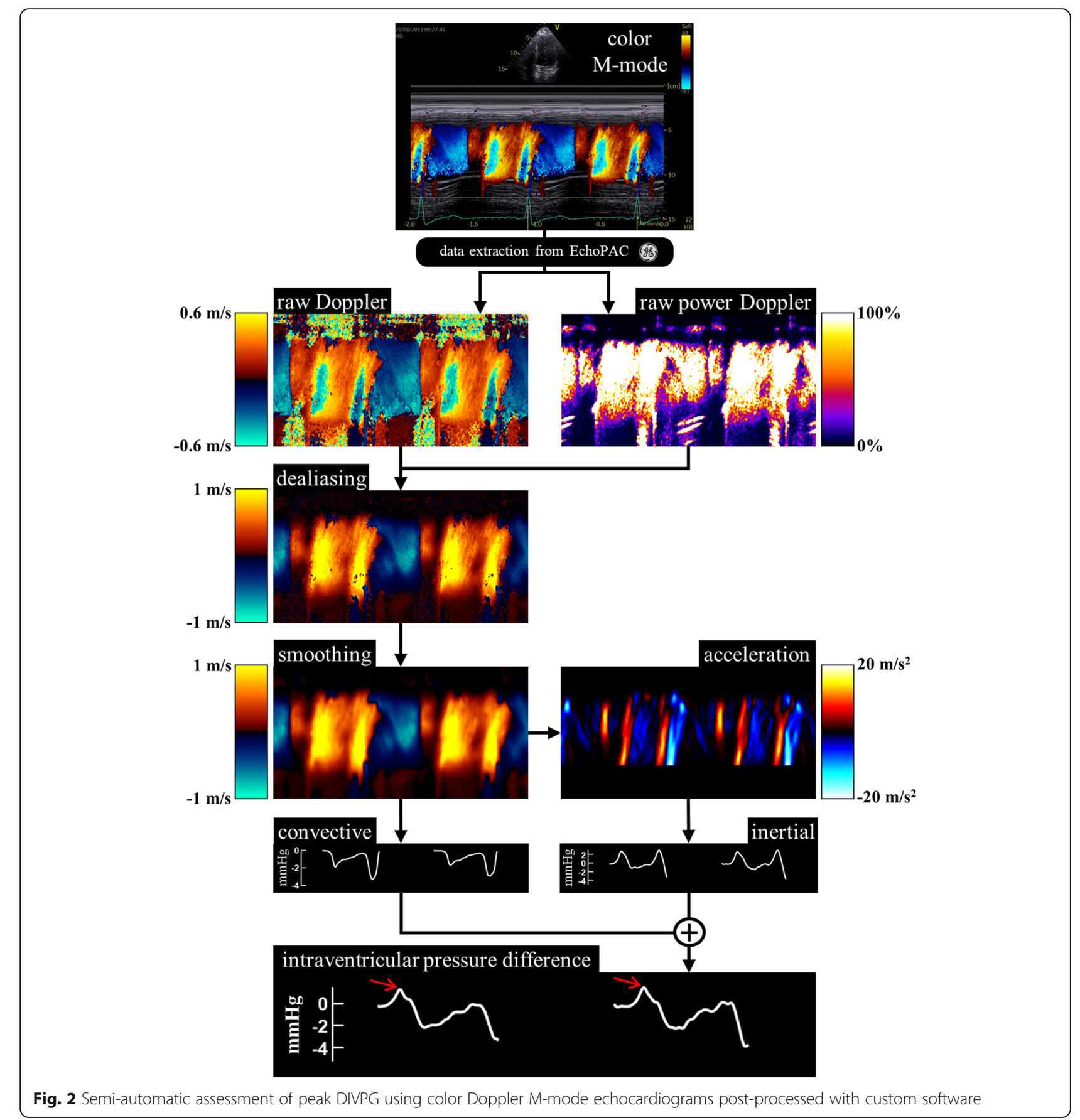

poor speckle-tracking quality of the LV endocardium in short-axis at the basal or apical level preventing untwisting rate assessment in 60/223 (27\%) subjects. DIVPG analysis was not feasible in only 9/223 (4\%) subjects because of either an important misalignment of Doppler beam with trans-mitral inflow [19] or an incorrect delimitation of LV basal and apical levels. The population of the study consisted of 32 patients scanned for cardiovascular risk assessment, and 122 healthy volunteers screened for non-specific symptoms or precompetitive physical exercise evaluation. The clinical characteristics of the population are summarized in Table 1 . Seventy-four percent of the participants had no medical history. LV morphological and functional echocardiographic parameters measured in our entire population are reported in Table 2. 
Table 1 Demographic and clinical parameters of the population $(n=154)$

\begin{tabular}{|c|c|c|c|}
\hline Parameters & Sample (\%) & Median $\left[25^{\mathrm{e}}-75^{\mathrm{e}}\right]$ & Range \\
\hline Male & $97(63)$ & & \\
\hline Age (yrs) & & $23[21-37.5]$ & $18-77$ \\
\hline Height (cm) & & 177 [170-183] & 126-206 \\
\hline Weight (kg) & & 82 [70.2-93.3] & $43.2-144$ \\
\hline Body mass index $\left(\mathrm{kg} / \mathrm{m}^{2}\right)$ & & $26.6[23.9-29.6]$ & $17.5-41.1$ \\
\hline Body surface area $\left(\mathrm{m}^{2}\right)$ & & $2[1.8-2.1]$ & $1.2-2.7$ \\
\hline Heart rate (bpm) & & $60.5[54-69]$ & $37-100$ \\
\hline Systolic blood pressure (mmHg) & & 120 [111-128] & $94-187$ \\
\hline Diastolic blood pressure (mmHg) & & $72[65-80]$ & $52-105$ \\
\hline Exercise training & $88(57.1)$ & & \\
\hline male & $58(65.9)$ & & \\
\hline age (yrs) & & $22[20-23]$ & $18-31$ \\
\hline Arterial hypertension & $20(13)$ & & \\
\hline male & $11(55)$ & & \\
\hline age (yrs) & & $53.5[47-61.5]$ & $22-77$ \\
\hline Diabetes & $4(2.6)$ & & \\
\hline Hyperlipidaemia & $7(4.5)$ & & \\
\hline \multicolumn{4}{|l|}{ Other medical history } \\
\hline immune disease & $5(3.2)$ & & \\
\hline Non cardiac transplantation & $2(1.3)$ & & \\
\hline Chronic viral infection & $2(1.3)$ & & \\
\hline
\end{tabular}

Values are expressed as median \pm interquartile $\left[25^{\mathrm{e}}-75^{\mathrm{e}}\right.$ percentile], and range

\section{Test-retest reproducibility of DIVPG acquisitions}

Test-retest reliability of DIVPG was determined in 20 subjects randomly selected from the echocardiographic database. For intra-observer reproducibility, the intraclass correlation coefficient was 0.97 [0.91-0.99], and the relative percentage difference was $4.28 \pm 4.52 \%$. For inter-observer reproducibility, the intraclass correlation coefficient was 0.97 [0.67-0.99], and the relative percentage difference was $8.96 \pm 5.28 \%$.

Relationships between early diastolic functional parameters There was a positive and significant correlation between peak DIVPG and peak untwisting rate $(\mathrm{r}=0.73 ; P<0.001)$ in our population (Fig. 3). This correlation was maintained regardless of age ( $\leq 50$ years old or $>50$ years old), gender, exercise training status, and personal history of hypertension (Fig. 4). Correlation between DIVPG and untwisting rate was slightly stronger in women compared to men (Fig. 4). Peaks E, e', Esr and Vp showed poor to moderate correlations with both peak DIVPG and peak untwisting rate (Figs. 5 and 6). Only peak DIVPG was inversely and significantly correlated with $\mathrm{E} / \mathrm{e}^{\prime}$ ratio. LA volume was not correlated with peak untwisting rate $(P=0.09)$. Multivariate analysis revealed that peak DIVPG was the only variable that was independently associated with peak untwisting rate $(\beta$-coefficient $=14.1 \pm 2$, $\mathrm{R}^{2}$-adjusted $\left.=0.53, P<0.0001\right)$.

\section{Clinical factors that influence untwisting rate and DIVPG}

Univariate analysis showed that peak untwisting rate significantly decreased with aging, that peak untwisting rate differed significantly between males and females (74.6 \pm $19.2 \mathrm{deg} / \mathrm{s}$ for females vs. $64.9 \pm 18.7 \mathrm{deg} / \mathrm{s}$ for males, $P=$ 0.002 ), and that peak untwisting rate was inversely associated with body mass index (Table 3). Multivariate analysis revealed that only age and sex were independently associated with peak untwisting rate (Table 3 ). As shown in Table 4, peak DIVPG was associated with age and chronic exercise training on univariate analysis, but age was the only variable that was independently associated with peak DIVPG on multivariate analysis. Peak DIVPG decreased with aging. There was no difference in peak DIVPG between men and women $(3.17 \pm 0.9 \mathrm{mmHg}$ vs. $3.21 \pm 1.1 \mathrm{mmHg}, P=0.81)$. The median age was similar between sexes (27 yrs. [22-47.5] for women vs. 23 yrs. [21-33] for men, $P=0.12$ ).

\section{Passive leg raising test}

During passive leg raising maneuver performed in 20 healthy male volunteers, we observed simultaneously 
Table 2 Echocardiographic analysis

\begin{tabular}{|c|c|c|c|}
\hline Variable & Mean \pm SD & Median $\left[25^{\mathrm{e}}-75^{\mathrm{e}}\right]$ & Range \\
\hline \multicolumn{4}{|l|}{ Morphology } \\
\hline LVIDd (mm) & $50.4 \pm 5.1$ & & $34-65$ \\
\hline LVIDd/BSA $\left(\mathrm{mm} / \mathrm{m}^{2}\right)$ & $25.6 \pm 3.1$ & & $17.7-39.5$ \\
\hline IVSd (mm) & $8.6 \pm 1.7$ & & $5-14$ \\
\hline PWTd (mm) & $8 \pm 1.4$ & & $5-11$ \\
\hline LV mass (g) & & 144 [115.3-168] & $70.6-219$ \\
\hline LV mass/BSA $\left(\mathrm{g} / \mathrm{m}^{2}\right)$ & & $70.1[61.1-78.8]$ & $43.5-108$ \\
\hline LA volume (ml) & $47.8 \pm 14.6$ & & $36-59$ \\
\hline LA volume/BSA (ml/m²) & $24.3 \pm 6.6$ & & $19.2-29$ \\
\hline LV ejection fraction (\%) & & $60[55-65]$ & $50-72$ \\
\hline LV global longitudinal strain (\%) & $19.6 \pm 2$ & & $15.3-25.3$ \\
\hline \multicolumn{4}{|l|}{ Diastolic function } \\
\hline \multicolumn{4}{|l|}{ Doppler } \\
\hline Peak transmitral E-wave $(\mathrm{cm} / \mathrm{s})$ & $72.2 \pm 13.6$ & & $33-105$ \\
\hline Peak transmitral A-wave $(\mathrm{cm} / \mathrm{s})$ & & 39 [32-48] & $22-97$ \\
\hline E/A ratio & $1.87 \pm 0.61$ & & $0.66-3.33$ \\
\hline Peak early diastolic tissue velocity e' $(\mathrm{cm} / \mathrm{s})$ & $12.8 \pm 2.9$ & & $5-18.5$ \\
\hline E/e' ratio & & $5.6[4.8-6.6]$ & $2.6-15$ \\
\hline \multicolumn{4}{|l|}{ Speckle tracking } \\
\hline Peak untwisting rate (deg/s) & $68.5 \pm 19.4$ & & $28.3-130$ \\
\hline Peak early diastolic strain rate $\mathrm{Esr}\left(\mathrm{s}^{-1}\right)$ & $1.45 \pm 0.32$ & & $0.67-1.62$ \\
\hline \multicolumn{4}{|l|}{ Color Doppler M-mode } \\
\hline Peak DIVPG (mmHg) & $3.2 \pm 1$ & & $1.6-7.7$ \\
\hline Peak Vp (cm/s) & $77.6 \pm 22.8$ & & $38-143$ \\
\hline ENp ratio & $0.99 \pm 0.3$ & & $0.3-1.7$ \\
\hline
\end{tabular}

Values are expressed as mean \pm SD if normally distributed or median and interquartile $\left[25^{\mathrm{e}}-75^{\mathrm{e}}\right.$ percentile]. BSA: body surface area, DIVPG diastolic intraventricular pressure gradients, IVS interventricular septal thickness in diastole, LA left atrial, LV left ventricle, LVIDd LV internal diameter in diastole, PWTd posterior wall thickness in diastole, $V p$ flow propagation velocity

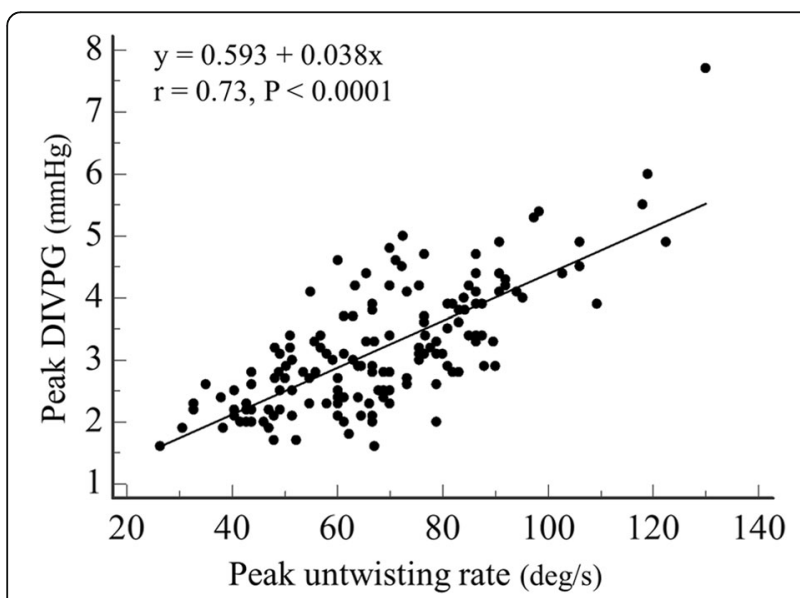

Fig. 3 Relationship between peak DIVPG and peak untwisting rate in the entire population significant variations of mean DIVPG $(2.71 \pm 0.77 \mathrm{mmHg}$ vs. $3.25 \pm 0.88 \mathrm{mmHg}, P<0.001)$ and untwisting rate $(65.5 \pm 14.2 \mathrm{deg} / \mathrm{s}$ vs. $77.1 \pm 20.5 \mathrm{deg} / \mathrm{s}, P=0.011)$, with inter-individual variability (Fig. 7). Correlations between untwisting rate and DIVPG were not significantly different before and after leg raising $(P=0.32)$. The heart rate was slightly but significantly increased after leg raising (65 [58-71] bpm vs. 68.5 [59-75] bpm, $P=0.03$ ).

\section{Discussion}

The present work has confirmed on a large adult population with preserved cardiac function a close relationship between the amplitudes of peak DIVPG and peak untwisting rate. This relationship remained strong regardless of age, gender, exercise training, chronic hypertension, and acute changes in LV load did not alter it. Although DIVPG is independently associated with untwisting rate and explains much of its variability, these two parameters of LV early diastolic function are complementary but not 


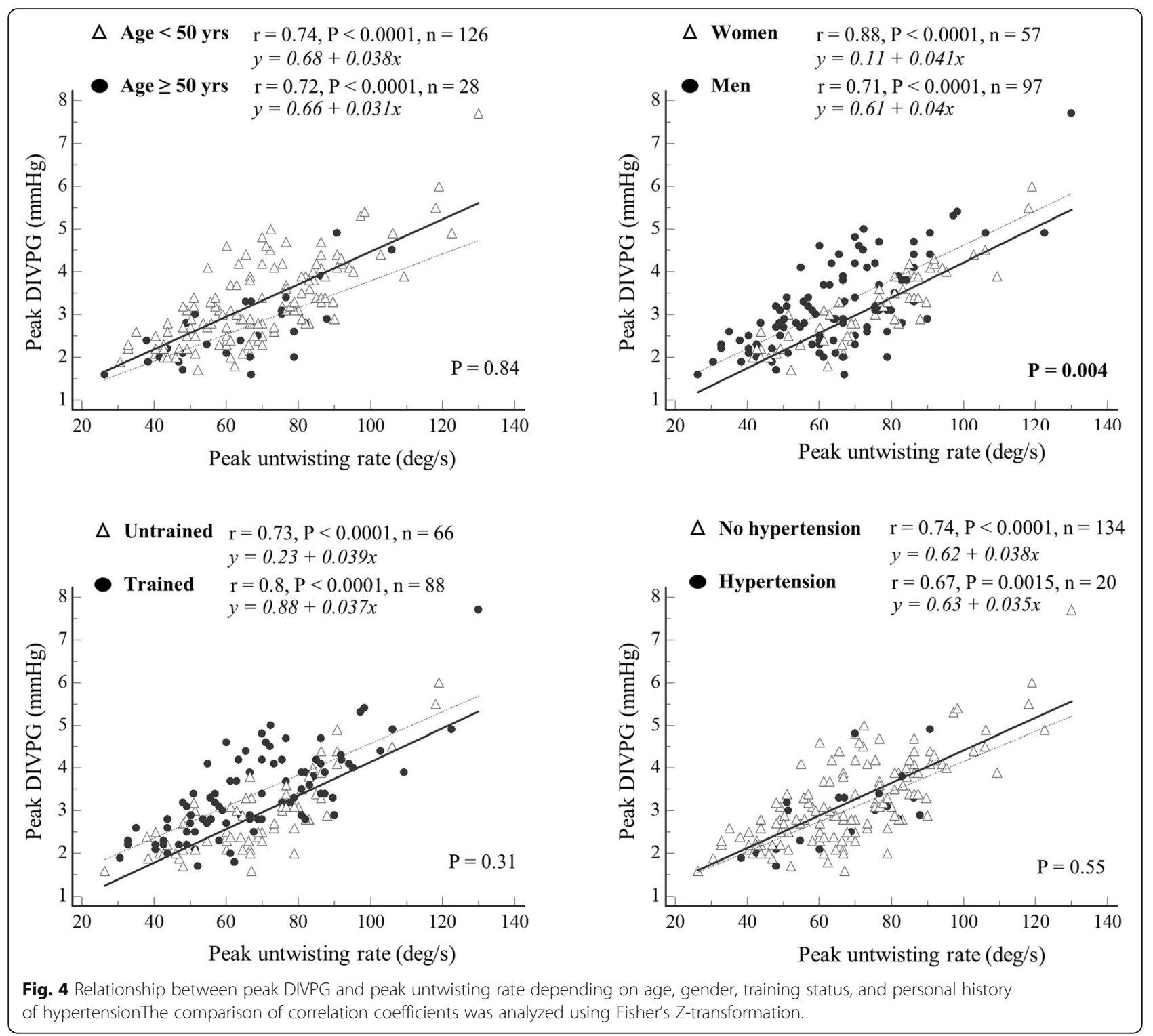

interchangeable as the former is related to blood flow and the latter to the myocardium.

DIVPG quantification by color Doppler M-mode provides a robust approach to assess early diastolic intraventricular inflow through the measure of the instantaneous velocity distribution. In contrast to the alias-based slope method for measuring $\mathrm{Vp}$ responsible for lower feasibility and reproducibility [9], which was weakly correlated with peak DIVPGs and untwisting rate, DIVPG assessment allows a more comprehensive evaluation of LV suction. Echocardiographic acquisition and calculation of DIVPGs are uncomplicated, it requires a single standard apical four-chamber view, and the use of automatic postprocessing ensures low test-retest variability and high technical feasibility, which was confirmed in the present study on a large adult cohort. Our software using velocity data before scan conversion could potentially be implemented on a work station and used in any clinical echo lab.

First human studies that have demonstrated the relationship between LV untwisting (assessed by tissue Doppler imaging) and DIVPGs were conducted on small groups of healthy volunteers $(n=20)$ and patients with hypertrophic cardiomyopathy $(n=7)[12,13]$. LV untwisting precedes DIVPG genesis which promotes LV suction work. In the temporal sequence of diastolic filling, these two mechanisms resulting from restoring forces precede peak early filling and LV expansion $[1,13]$. In line with these observations, our study confirmed in a large adult cohort without heart disease a similar good correlation between the magnitudes of peak DIVPG and peak untwisting rate assessed by speckle tracking. Our multivariate analysis showed that peak DIVPG was independently 

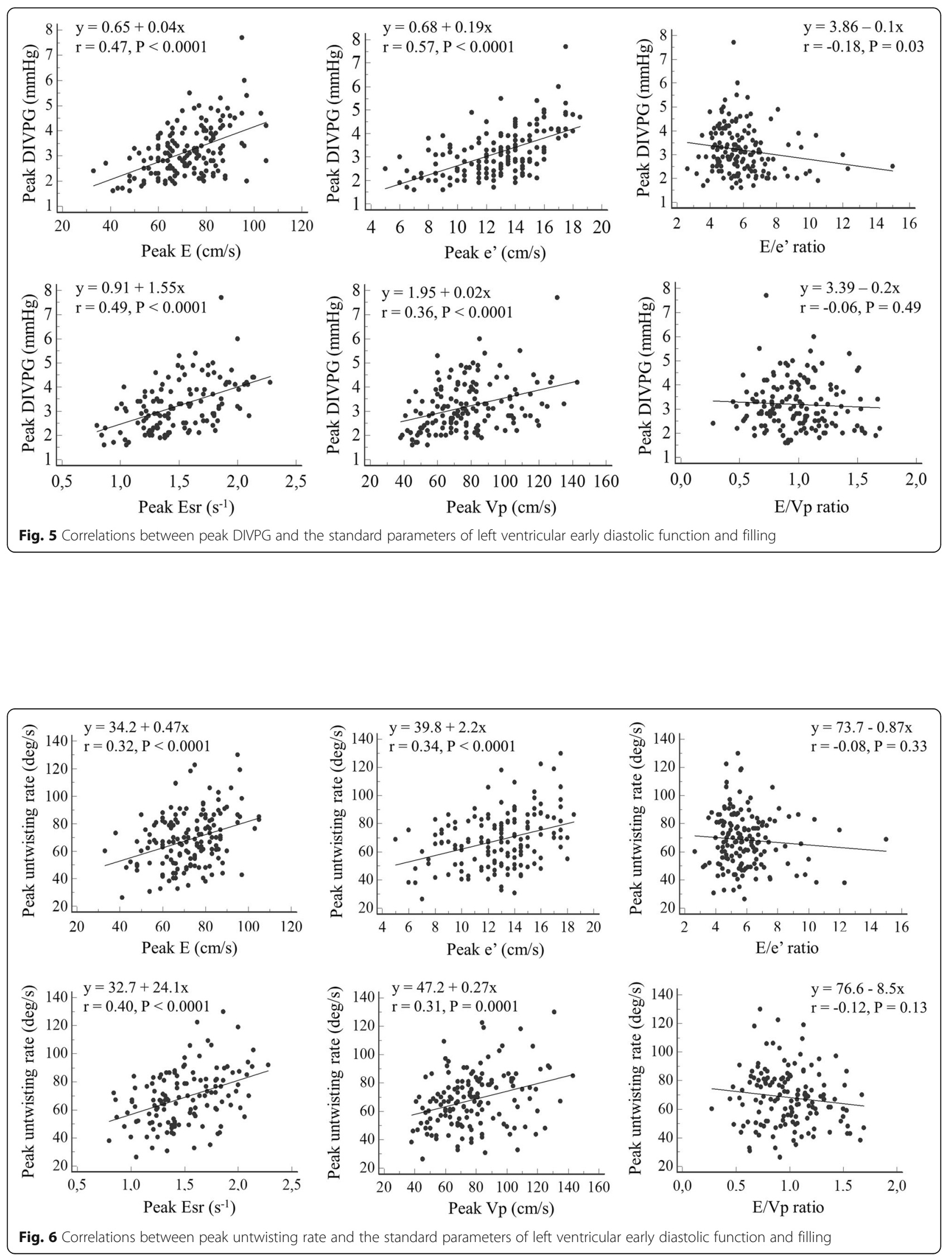
Table 3 Results of univariate and stepwise multivariate analysis for untwisting rate

\begin{tabular}{|c|c|c|c|c|c|c|c|c|}
\hline \multirow[t]{2}{*}{ Variable } & \multicolumn{4}{|l|}{ Univariate analysis } & \multicolumn{4}{|l|}{ Multivariate analysis } \\
\hline & Coefficient (Slope) & Std Error & $r_{\text {partial }}$ & $P$ & Coefficient (Slope) & Std Error & $r_{\text {partial }}$ & $P$ \\
\hline Age & -0.21 & 0.1 & -0.16 & $0.05^{*}$ & -0.25 & 0.1 & -0.2 & 0.015 \\
\hline Sex & -9.7 & 3.1 & -0.24 & $0.002^{*}$ & -11.2 & 3.1 & -0.28 & 0.0005 \\
\hline Body mass index & -0.69 & 0.32 & -0.17 & $0.03^{*}$ & -0.43 & 0.32 & -0.11 & 0.19 \\
\hline Heart rate & 0.064 & 0.13 & 0.04 & 0.62 & & & & \\
\hline Systolic blood pressure & 0.11 & 0.11 & 0.08 & 0.34 & & & & \\
\hline Diastolic blood pressure & 0.042 & 0.15 & 0.02 & 0.78 & & & & \\
\hline Exercise training & 0.14 & 3.2 & 0.004 & 0.96 & & & & \\
\hline
\end{tabular}

* Variables with a P-value $<0.1$ in univariate analysis were included in the multivariate analysis

associated with peak untwisting rate. Due to this strong interplay between DIVPG and untwisting rate, DIVPG could provide a reliable ( $96 \%$ of feasibility versus only $73 \%$ for untwisting rate) and less time-consuming (one apical acquisition automatically post-processed) evaluation of LV early diastolic recoil. Peak untwisting rate was shown to be associated not only with LV relaxation rate but also with LV restoring forces [5]. Studies on intraventricular pressure gradients have also confirmed a close relationship with both LV relaxation rate and contractility $[3,4,20]$. Significant but weaker correlations for untwisting rate and DIVPGs were observed with early-diastolic filling velocity (assessed by E) and myocardial lengthening velocity (assessed by e' and Esr). Although LV filling is related to many factors, including left atrial function and venous return, the association between LV restoring forces and early filling could be expected through LV suction $[1,12$, 13]. LV early-diastolic lengthening velocity was shown to be determined by LV suction, in addition to LV lengthening load and stiffness [21]. Nonetheless, e' and Esr are region-dependent parameters, which limits the assessment of the LV base-apex velocity gradient.

In this study, age similarly affected the untwisting rate and DIVPG. We found that age was an independent predictor for both peak untwisting rate and peak DIVPG. Therefore, the relationship between untwisting rate and DIVPG remained similar in younger and older subgroups. Impairment in LV diastolic function is part of the aging process of the heart, which affects myocardial intrinsic relaxation proprieties and disturbs cellular calcium homeostasis [22]. Previous works have demonstrated that untwisting rate is delayed with aging, which in turn affects LV active early filling and intraventricular pressure gradients [23, 24]. Our results confirmed a decrease in peak untwisting rate and peak DIVPG with aging. The relationship between untwisting rate and DIVPG was slightly but significantly better in women compared to men. In our population, male sex seemed to independently and negatively affect peak untwisting rate, the latter being greater in women. Previous observations have reported that females exhibited greater LV rotation and faster untwisting velocity following acute preload reduction and inotropy stimulation [25], suggesting an adaptive mechanism to generate adequate intraventricular pressure gradients and filling and to balance gender-related differences in LV geometric and myocardial intrinsic functional proprieties [26].

Previous observations in healthy volunteers have suggested that the load dependency of LV untwisting rate may be a physiological compensatory mechanism to minimize the effects of load variations on LV filling and stroke volume [27, 28]. Experimental findings have confirmed that acute changes in LV early diastolic load have direct effects on untwisting rate, in addition to LV relaxation rate and restoring forces [5]. Popovic et al. [29] showed that variations in DIVPGs assessed by color

Table 4 Results of univariate and stepwise multivariate analysis for diastolic intraventricular pressure gradients

\begin{tabular}{|c|c|c|c|c|c|c|c|c|}
\hline \multirow[t]{2}{*}{ Variable } & \multicolumn{4}{|l|}{ Univariate analysis } & \multicolumn{4}{|l|}{ Multivariate analysis } \\
\hline & Coefficient (Slope) & Std Error & $r_{\text {partial }}$ & $P$ & Coefficient (Slope) & Std Error & $r_{\text {partial }}$ & $P$ \\
\hline Age & -0.02 & 0.01 & -0.36 & $<0.0001^{*}$ & -0.24 & 0.005 & -0.36 & $<0.0001$ \\
\hline Sex & 0.04 & 0.17 & 0.02 & 0.81 & & & & \\
\hline Body mass index & 0.014 & 0.017 & 0.07 & 0.4 & & & & \\
\hline Heart rate & -0.006 & 0.007 & -0.07 & 0.37 & & & & \\
\hline Systolic blood pressure & 0.002 & 0.006 & 0.03 & 0.74 & & & & \\
\hline Diastolic blood pressure & -0.01 & 0.008 & -0.11 & 0.17 & & & & \\
\hline Exercise training & 0.51 & 0.16 & 0.25 & $0.002^{*}$ & -0.03 & 0.22 & -0.01 & 0.89 \\
\hline
\end{tabular}

* Variables with a P-value $<0.1$ in univariate analysis were included in the multivariate analysis 

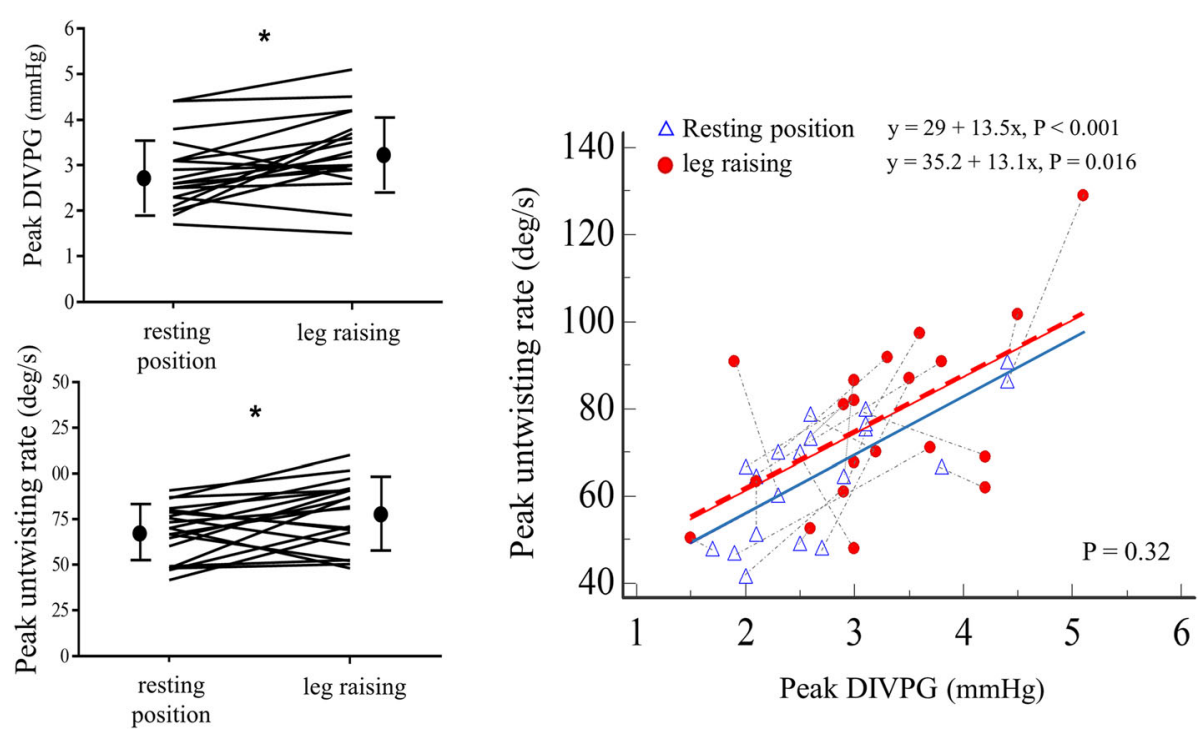

Fig. 7 Relationship between DIVPG and untwisting rate during a passive leg raising maneuver. Mean peaks DIVPG and untwisting rate increased significantly after a passive leg raising in 20 healthy volunteers (left, ${ }^{*} P<0.001$ ), without altering the correlation between the magnitudes of peak untwisting rate and peak DIVPG (right). Each pair of resting and leg raising states was connected by a line

Doppler M-mode observed during acute load manipulations were related to changes in pulmonary capillary wedge pressure and were modulated by LV relaxation rate. We used passive leg raising as a simple and safety maneuver to evaluate the effects of a transient cardiac load variation on the relationship between untwisting rate and DIVPG. Our results are consistent with a load dependency of these two parameters assessed by echocardiography, without any significant change observed in their relationship.

\section{Limitations}

The first limitation is related to the techniques used for non-invasive assessment of intraventricular pressure gradients and untwisting rate. Since color Doppler is a onedimensional imaging technique (only velocity vector projections are returned), flow needs to be coaxially interrogated by the ultrasound M-mode scan-line so as not to underestimate blood velocity. Thus, we cannot preclude some misalignment in the course of this study. However, based on our past experience with this approach, the DIVPG calculation is only marginally or not affected by a slight misalignment of the Doppler scanline with respect to the flow streamline [19]. Moreover, although special care was taken to ensure that the peak untwisting rate was measured as previously described, we did not assess the exact time of opening of the mitral valve [7]. Secondly, passive leg raising is commonly used to increase cardiac preload. It is also known to influence central blood pressure via baroreceptor activation in healthy subjects [30]. We did not measure the early diastolic load increment nor the potential effects of sympathetic activation that might have led to some of the inter-individual variability in the untwisting rate and DIVPG measurements. We cannot also predict whether the effects of passive leg raising would be similar in females. Untwisting rate response to preload reduction was shown to be greater in females than males but with no significant difference following preload augmentation by saline infusion [25]. Finally, our trained population was much younger than sedentary subjects. With the understanding of the determinant effect of age on both DIVPGs and untwisting rate that we have shown, this selection bias might explain that chronic exercise training was not associated with parameters of LV active relaxation in our study.

\section{Conclusions}

DIVPG measured by color Doppler M-mode was well correlated and independently associated with LV untwisting rate. Their interdependence indicates a strong physiological coupling between the intraventricular fluid forces and the myocardium relaxation. This echocardiographic approach to DIVPG estimation, highly feasible and reproducible, could improve the assessment of LV diastolic function. Our team is currently working on the development of turnkey clinical software for computing DIVPG, to further study its diagnostic value in patients with heart diseases.

\section{Abbreviations}

A: Peak velocity of late filling wave; DIVPG: Diastolic intraventricular pressure gradient.; E: Peak velocity of early filling wave.; e': Mitral annulus early peak velocity:; Esr: Peak of global early diastolic strain rate.; GLS: Global Iongitudinal strain.; LA: Left atrial.; LV: Left ventricle.; LVEF: Left ventricular ejection fraction.; Vp: Color M-mode Doppler flow propagation velocity. 


\section{Acknowledgments}

Authors thank the University of Montreal Hospital Research Center.

\section{Authors' contributions}

$A H, D G, P M, H N$, and FT conceived and designed the study. AH, AE and FT performed the acquisitions. AH, DG, ES, PABR, JDT, and FT analyzed and interpreted data. DG created the software. AH, DG and FT drafted the manuscript. AH, DG, ES, PABR, AE, JDT, PM, HN, FT participated in the revision of the manuscript. All authors read and approved the final manuscript.

\section{Funding}

None.

\section{Availability of data and materials}

The datasets used and/or analyzed during the current study are available from the corresponding author on reasonable request.

\section{Ethics approval and consent to participate}

The study was approved by the research ethics committee of the Research Center of the University Hospital of Montreal (2019-8217 / 18.348), in addition to the consent of the participants to be enrolled in the research database (2013-3966 / CE 12.029).

\section{Consent for publication}

\section{Not applicable.}

\section{Competing interests}

The authors declare that they have no competing interests.

\section{Author details}

'Department of Clinical Physiology, INSERM COMETE, Normandie Univ, UNICAEN, CHU de Caen Normandie, 14000 Caen, France. ${ }^{2}$ Department of Cardiology, Normandie Univ, UNICAEN, CHU de Caen Normandie, 14000 Caen, France. ${ }^{3}$ Research Center of the Hospital of the University of Montreal (Centre de Recherche du Centre Hospitalier de l'Université de Montréal), Montreal, Canada. ${ }^{4}$ CREATIS, CNRS UMR 5220, INSERM U1206, Université Lyon 1, INSA Lyon, Villeurbanne, France. ${ }^{5}$ Department of Cardiology, Bluhm Cardiovascular Institute, Northwestern University, Chicago, USA.

\section{Received: 23 December 2019 Accepted: 7 February 2020}

\section{Published online: 19 February 2020}

\section{References}

1. Rademakers FE, Buchalter MB, Rogers WJ, Zerhouni EA, Weisfeldt ML, Weiss $J \mathrm{~L}$, et al. Dissociation between left ventricular untwisting and filling Accentuation by catecholamines. Circulation. 1992:85:1572-81.

2. Courtois M, Kovács SJ, Ludbrook PA. Transmitral pressure-flow velocity relation. Importance of regional pressure gradients in the left ventricle during diastole. Circulation. 1988;78:661-71.

3. Firstenberg MS, Smedira NG, Greenberg NL, Prior DL, McCarthy PM, Garcia MJ, et al. Relationship between early diastolic intraventricular pressure gradients, an index of elastic recoil, and improvements in systolic and diastolic function. Circulation. 2001;104:1330-5.

4. Firstenberg MS, Greenberg NL, Garcia MJ, Thomas JD. Relationship between ventricular contractility and early diastolic intraventricular pressure gradients: a diastolic link to systolic function. J Am Soc Echocardiogr. 2008;21:501-6.

5. Opdahl A, Remme EW, Helle-Valle T, Edvardsen T, Smiseth OA. Myocardial relaxation, restoring forces, and early-diastolic load are independent determinants of left ventricular untwisting rate. Circulation. 2012;126:1441-51

6. Helle-Valle T, Crosby J, Edvardsen T, Lyseggen E, Amundsen BH, Smith $\mathrm{H}-\mathrm{J}$, et al. New noninvasive method for assessment of left ventricular rotation: speckle tracking echocardiography. Circulation. 2005;112: 3149-56.

7. Notomi Y, Lysyansky P, Setser RM, Shiota T, Popović ZB, Martin-Miklovic MG, et al. Measurement of ventricular torsion by two-dimensional ultrasound speckle tracking imaging. J Am Coll Cardiol. 2005:45:2034-41.

8. Kim H-K, Sohn D-W, Lee S-E, Choi S-Y, Park J-S, Kim Y-J, et al. Assessment of left ventricular rotation and torsion with two-dimensional speckle tracking echocardiography. J Am Soc Echocardiogr. 2007;20:45-53.
9. Nagueh SF, Smiseth OA, Appleton CP, Byrd BF, Dokainish H, Edvardsen T, et al. Recommendations for the evaluation of left ventricular diastolic function by echocardiography: an update from the American Society of Echocardiography and the European Association of Cardiovascular Imaging. Eur Heart J Cardiovasc Imaging. 2016;17:1321-60.

10. Firstenberg MS, Vandervoort PM, Greenberg NL, Smedira NG, McCarthy PM, Garcia MJ, et al. Noninvasive estimation of transmitral pressure drop across the normal mitral valve in humans: importance of convective and inertial forces during left ventricular filling. J Am Coll Cardiol. 2000;36:1942-9.

11. Greenberg NL, Vandervoort PM, Firstenberg MS, Garcia MJ, Thomas JD. Estimation of diastolic intraventricular pressure gradients by Doppler Mmode echocardiography. Am J Physiol Heart Circ Physiol. 2001;280: H2507-15.

12. Notomi Y, Martin-Miklovic MG, Oryszak SJ, Shiota T, Deserranno D, Popovic $Z B$, et al. Enhanced ventricular untwisting during exercise: a mechanistic manifestation of elastic recoil described by Doppler tissue imaging. Circulation. 2006;113:2524-33.

13. Notomi Y, Popovic ZB, Yamada H, Wallick DW, Martin MG, Oryszak SJ, et al. Ventricular untwisting: a temporal link between left ventricular relaxation and suction. Am J Physiol Heart Circ Physiol. 2008;294: H505-13.

14. Lang RM, Badano LP, Mor-Avi V, Afilalo J, Armstrong A, Ernande L, et al. Recommendations for cardiac chamber quantification by echocardiography in adults: an update from the American Society of Echocardiography and the European Association of Cardiovascular Imaging. J Am Soc Echocardiogr. 2015;28:1-39 e14.

15. Bermejo J, Antoranz JC, Yotti R, Moreno M, García-Fernández MA. Spatiotemporal mapping of intracardiac pressure gradients. A solution to Euler's equation from digital postprocessing of color Doppler M-mode echocardiograms. Ultrasound Med Biol. 2001;27:621-30.

16. Garcia D, Kadem L, Savéry D, Pibarot P, Durand L-G. Analytical modeling of the instantaneous maximal transvalvular pressure gradient in aortic stenosis. J Biomech. 2006:39:3036-44.

17. Muth S, Dort S, Sebag IA, Blais M-J, Garcia D. Unsupervised dealiasing and denoising of color-Doppler data. Med Image Anal. 2011;15:577-88.

18. Garcia D. Robust smoothing of gridded data in one and higher dimensions with missing values. Comput Stat Data Anal. 2010;54:1167-78.

19. Greenberg N, Krucinski S, Thomas J. Significance of color Doppler M-mode Scanline orientation in the non-invasive assessment of Intraventricular pressure gradients. Comput Cardiol. 1997:605-8.

20. Yotti R, Bermejo J, Benito Y, Antoranz JC, Desco MM, Rodríguez-Pérez D, et al. Noninvasive estimation of the rate of relaxation by the analysis of intraventricular pressure gradients. Circ Cardiovasc Imaging. 2011;4:94-104

21. Opdahl A, Remme EW, Helle-Valle T, Lyseggen E, Vartdal T, Pettersen E, et al. Determinants of left ventricular early-diastolic lengthening velocity: independent contributions from left ventricular relaxation, restoring forces, and lengthening load. Circulation. 2009;119:2578-86.

22. Oxenham H, Sharpe N. Cardiovascular aging and heart failure. Eur J Heart Fail. 2003;5:427-34.

23. Maufrais C, Schuster I, Doucende G, Vitiello D, Rupp T, Dauzat M, et al. Endurance training minimizes age-related changes of left ventricular twistuntwist mechanics. J Am Soc Echocardiogr. 2014;27:1208-15.

24. Zhang $Y$, Zhou Q, Pu D, Zou L, Tan Y. Differences in left ventricular twist related to age: speckle tracking echocardiographic data for healthy volunteers from neonate to age 70 years. Echocardiogr. 2010:27:1205-10.

25. Williams AM, Shave RE, Stembridge M, Eves ND. Females have greater left ventricular twist mechanics than males during acute reductions to preload. Am J Physiol Heart Circ Physiol. 2016;311:H76-84.

26. Hayward CS, Kalnins WW, Kelly RP. Gender-related differences in left ventricular chamber function. Cardiovasc Res. 2001:49:340-50.

27. Weiner RB, Weyman AE, Khan AM, Reingold JS, Chen-Tournoux AA, Scherrer-Crosbie M, et al. Preload dependency of left ventricular torsion: the impact of normal saline infusion. Circ Cardiovasc Imaging. 2010;3:672-8.

28. Hodt A, Hisdal J, Stugaard M, Stranden E, Atar D, Steine K. Increased LV apical untwist during preload reduction in healthy humans: an echocardiographic speckle tracking study during lower body negative pressure. Physiol Rep. 2015;3(3):e12330.

29. Popović ZB, Prasad A, Garcia MJ, Arbab-Zadeh A, Borowski A, Dijk E, et al. Relationship among diastolic intraventricular pressure gradients, relaxation, 
and preload: impact of age and fitness. Am J Physiol Heart Circ Physiol. 2006;290:H1454-9.

30. Kamran H, Salciccioli L, Gusenburg J, Kazmi H, Ko EH, Qureshi G, et al. The effects of passive leg raising on arterial wave reflection in healthy adults. Blood Press Monit. 2009;14:202-7.

\section{Publisher's Note}

Springer Nature remains neutral with regard to jurisdictional claims in published maps and institutional affiliations.

Ready to submit your research? Choose BMC and benefit from:

- fast, convenient online submission

- thorough peer review by experienced researchers in your field

- rapid publication on acceptance

- support for research data, including large and complex data types

- gold Open Access which fosters wider collaboration and increased citations

- maximum visibility for your research: over $100 \mathrm{M}$ website views per year

At $\mathrm{BMC}$, research is always in progress. 